\title{
Analytical and numerical studies of fluid instabilities in relativistic jets.
}

\author{
M. Perucho ${ }^{1}$, A.P. Lobanov ${ }^{1}$ and J.M. ${ }^{\text {Martí }}{ }^{2}$ \\ 1 Max-Planck-Institut für Radioastronomie. Auf dem Hügel 6953121 Bonn, \\ Germany. perucho@mpifr-bonn.mpg.de alobanov@mpifr-bonn.mpg.de \\ 2 Departament d'Astronomia i Astrofísica. Universitat de València. C/ Dr. \\ Moliner 5046100 Burjassot, València, Spain. jose-maria.marti@uv.es
}

\begin{abstract}
Summary. Relativistic outflows represent one of the best-suited tools to probe the physics of AGN. Numerical modelling of internal structure of the relativistic outflows on parsec scales provides important clues about the conditions and dynamics of the material in the immediate vicinity of the central black holes in AGN. We investigate possible causes of the structural patterns and regularities observed in the parsecscale jet of the well known quasar $3 \mathrm{C} 273$. We compare the model with the radio structure observed in 3C 273 on parsec scales using very long baseline interferometry (VLBI) and constrain the basic properties of the flow. Our results show that KelvinHelmholtz instabilities are the most plausible mechanism to generate the observed structures.
\end{abstract}

\section{Introduction}

3C 273 is the brightest quasar known. Its relative proximity $(z=0.158,[1])$ has made it a paradigmatic object studied throughout the entire spectral range [2]. One of the most impressive features of 3C 273 is an apparently onesided relativistic outflow emanating from the quasar nucleus [2] and extending up to a deprojected distance of about $60 \mathrm{kpc}$. The jet in $3 \mathrm{C} 273$ has been observed on parsec scales using ground and space VLBI $[3,4,5,6]$. Space VLBI observations made at $5 \mathrm{GHz}$ with the $\mathrm{VSOP}^{3}$ revealed a double helical patterns inside the parsec-scale jet in 3C 273 [5]. Linear perturbation analysis of Kelvin-Helmholtz (K-H) instability [7] applied to model these structures yielded an accurate estimate of the bulk parameters of the flow [5]. A different interpretation suggests that a helical magnetic field could generate such a structure [6]. We study the first possibility. A bulk Lorentz factor $W=2.1$ obtained in [5] is below the values inferred from superluminal motions observed in the jet $(W=5-10,[4])$, which suggests that the K-H instability develops in a slower underlying flow, while the fast components are shock waves inside the

\footnotetext{
${ }^{3}$ VLBI Space Observatory Program
} 
jet. Four K-H modes were found acting on parsec scales in the jet in $3 \mathrm{C} 273$ : the second helical body mode with a wavelength of 2 mas, the first elliptical and helical body modes, both with wavelengths close to 4 mas, and the elliptical surface mode, with a wavelength of 12 mas. Additionally a structure with a wavelength of 18 mas was interpreted as an externally driven helical surface mode. Our previous works $[8,9]$ have shown that numerical simulations can be used effectively to connect the linear perturbation analysis with studies of non-linear regime of $\mathrm{K}-\mathrm{H}$ instability. Our aim here is to use the jet parameters determined in [5] as initial conditions for numerical RHD simulations of a perturbed jet and compare the numerical results with the observed structures.

\section{Numerical simulations}

\subsection{Simulation 3C 273-A}

We start with a steady jet with a Lorentz factor $W=2.1$, a density contrast with the external medium $\eta=0.023$, a sound speed $c_{s, j}=0.53 c$ in the jet and $c_{s, a}=0.08 c$ in the external medium, and the perfect gas equation of state (with adiabatic exponent $\gamma=4 / 3$ ). Assuming an angle to the line of sight $\theta=15^{\circ}$, the observed jet extends up to $\approx 170 \mathrm{pc}$. With the jet radius $R_{\mathrm{j}}=0.8 \mathrm{pc}$ [5], the numerical grid covers $211 R_{\mathrm{j}}($ axial $) \times 8 R_{\mathrm{j}} \times 8 R_{\mathrm{j}}$ (transversal), i.e., $169 \mathrm{pc} \times 6.4 \mathrm{pc} \times 6.4 \mathrm{pc}$. The resolution is 16 cells $/ R_{\mathrm{j}}$ in the transversal direction and 4 cells $/ R_{\mathrm{j}}$ in the direction of the flow. A shear layer of $2 R_{\mathrm{j}}$ in width is included in the initial rest mass density and axial velocity profiles to keep numerical stability of the initial jet. Elliptical and helical modes are induced at the inlet.

Frequencies of the excited modes are computed from the observed wavelengths of modes, $\lambda^{\text {obs }}$, corrected for projection effects, relativistic bulk motion and wave pattern speed, $v_{w}=0.23 c$, according to $\omega=2 \pi v_{w} / \lambda^{\text {theor }}$, where

$$
\lambda^{\text {theor }}=\frac{\lambda^{\mathrm{obs}}\left(1-v_{w} / c \cos \theta\right)}{\sin \theta},
$$

Three wavelengths have been identified in the simulation: a helical $\lambda_{1}^{\mathrm{sim}}=4 R_{\mathrm{j}}$ perturbation, a helical $\lambda_{2}^{\text {sim }}=25 R_{\mathrm{j}}$ perturbation and an elliptical $\lambda_{3}^{\text {sim }}=50 R_{\mathrm{j}}$ perturbation. The wave speeds $v_{w, 2} \simeq 0.38 c$ and $v_{w, 3} \simeq 0.2 c$ are estimated for the $\lambda_{2}^{\text {sim }}$ and $\lambda_{3}^{\text {sim }}$ modes, respectively. An upper limit for $v_{w, 1}$ is given by the flow speed $(0.88 c)$. With these wave speeds, $\lambda_{1}^{\text {sim }}$ would be observed as a 2.27 mas structure, $\lambda_{2}^{\text {sim }}$ as a 3.37 mas structure and $\lambda_{3}^{\text {sim }}$ as a 5.5 mas structure. These are similar to the shorter-wavelength structures found in the observations (2.27 mas vs 2 mas, 3.37 and 5.5 mas vs 4 mas). It remains to be studied why the simulations do not reproduce readily the longer modes. The longest mode, with the wave speed given in [5], corresponds to a simulated wavelength of $150 R_{\mathrm{j}}$, which is difficult to reproduce even in the grid of this simulation, in particular when shorter harmonics grow fast and disrupt the 
flow. The disruptive evolution may be caused by a number of factors, including the absence of a stabilizing magnetic field in the simulations.

\subsection{Simulation 3C 273-B}

In the second simulation, we try to study the effect of precession and injection of fast components on the observed structures in the jet. The precession frequency is given by the observed $15 \mathrm{yr}$ periodicity of the position angle variations in the inner jet ([4]). The frequency of ejections of components is set by the reported 1 yr periodicity ([3]). The duration of each ejection is estimated to be 2 months, from the approximate inspiralling time from an orbit at $\sim 6 R_{G}$ around a $5.510^{8} M_{\odot}$. Velocity of the components is taken as constant, with the mean value of those given in [4], i.e., Lorentz factor $W \sim 5$. The components are treated as shells of diameter $0.5 R_{\mathrm{j}}$ ejected at the center of the transversal grid. The numerical grid for this simulation covers $30 R_{\mathrm{j}}$ (axial) $\times 6 R_{\mathrm{j}} \times 6 R_{\mathrm{j}}$ (transversal), i.e., $24 \mathrm{pc} \times 4.8 \mathrm{pc} \times 4.8 \mathrm{pc}$. The resolution of the grid is 16 cells $/ R_{\mathrm{j}}$ in both transversal directions and 32 cells $/ R_{\mathrm{j}}$ in the direction of the flow.

We find two structures in the simulation: a pinching perturbation with a wavelength of $0.4 R_{\mathrm{j}}$ caused by the injection of components, and a helical perturbation with a wavelength of $4.0 R_{\mathrm{j}}$ associated with the precession. The wave speed associated with these structures is $\leq 0.98 c$. This upper limit for the wave speed results in observed wavelengths (from Eq. 1) of 0.6 and 6 mas, much smaller than the 2-4 mas and 18 mas expected from the observations. This results further supports the identification of the shorter modes with K-H instability modes. We also find that the $15 \mathrm{yr}$ precession period cannot account for the observed longer, 18-mas wavelength. Thus, either the longer-wavelength structure is driven by a different mechanism, or the $15 \mathrm{yr}$ periodicity in the source is not associated with the precession.

\section{References}

1. M.A. Strauss, J.P. Huchra, M. Davies, et al.: ApJSS 83, 29 (1992)

2. T. J.-L. Courvoisier: A\&ARv 9, 1 (1998)

3. T.P. Krichbaum, A. Witzel, J.A. Zensus: From centimetre to millimetre wavelengths: A high angular resolution study of 3C273. In: Proc. of the 5th EVN Symposium, ed by J.E. Conway, A.J. Polatidis, R.S. Booth, Y.W. Pihlström (Onsala Space Observatory 2000) pp 25

4. Z. Abraham, E.A. Carrara, J.A. Zensus, S.C. Unwin: A\&AS 115, 543 (1996)

5. A.P. Lobanov, J.A. Zensus: Science 294, 128 (2001)

6. K. Asada, M. Inoue, Y. Uchida, et al.: PASJ 54, L39 (2002)

7. P.E. Hardee: ApJ 533, 176 (2000)

8. M.P. Perucho, M. Hanasz, J.M. Martí, H. Sol: A\&A 427, 415 (2004)

9. M.P. Perucho, J.M. Martí, M. Hanasz: A\&A 427, 431 (2004) 